\title{
Long non-coding RNA BX357664 inhibits cell proliferation and metastasis in human lung cancer
}

\author{
SHU-HUI XIAO ${ }^{1}$, GONG-XIANG LI $^{1}$ and LINGLI QUAN ${ }^{2}$ \\ ${ }^{1}$ Department of Clinical Laboratory Medicine, The People's Hospital of Jinyi, Jinyi, Shanxi 710003; \\ ${ }^{2}$ The First Department of Respiratory of Central Hospital of Zhuzhou, Zhuzhou, Hunan 412000;
}

Received December 19, 2016; Accepted June 18, 2018

DOI: $10.3892 / \mathrm{ol} .2019 .9886$

\begin{abstract}
Long non-coding RNAs (lncRNAs) have been investigated in human carcinogenesis. The lncRNA BX357664 has emerged as a novel lncRNA that was initially recognized by a microarray analysis. The present study aimed to identify the expression and functional roles of IncRNA BX357664 in lung cancer. The transcription level of BX357664 was initially revealed to be downregulated in clinical lung cancer tissues and in a series of lung cancer cell lines. Clinical data demonstrated that the high expression of BX357664 was associated with tumor size, distant metastasis and Tumor-Node-Metastasis stage. Following the overexpression of BX357664 in A549 and 95D cells, the potential of cells to form colonies, as well as the proliferation and motility abilities, were revealed to be decreased. Furthermore, the cell cycle was arrested in the G0/G1 phase by BX357664 modulation. Transwell analysis and a wound-healing assay also demonstrated that overexpression of BX357664 in A549 and 95D cells significantly inhibited cell migration and invasion. These data suggested that BX357664 inhibits cell proliferation and metastasis in lung cancer. The results of the present study provided evidence that BX357664 is a novel lncRNA that may aid in the diagnosis and treatment of lung cancer.
\end{abstract}

\section{Introduction}

Lung cancer is one of the most prevalent types of malignancy worldwide, ranking as the first and second leading causes of cancer-associated mortality in males and females, respectively (1). Based on a cancer statistic in 2013, there were 228,190 newly diagnosed cases of lung cancer, which consisted of 118,080 males and 110,110 females in the United States of America. Among these cases, it was estimated that 87,260 male

Correspondence to: Dr Lingli Quan, The First Department of Respiratory of Central Hospital of Zhuzhou, 116 South Changjiang Road, Zhuzhou, Hunan 412000, P.R. China

E-mail: jing1969wang@sina.com

Key words: BX357664, lung cancer, proliferation, metastasis
(73.9\%) and 72,220 female (65.6\%) patients succumbed to this malignancy (2). Therefore, this serious situation mandates the necessity to identify novel therapeutic targets for the clinical diagnosis and treatment of lung cancer.

Recently, high-throughput transcriptome analysis has revealed that $>90 \%$ of the transcriptome is transcribed into non-coding RNAs, among which long non-coding RNAs (lncRNAs) have been implicated in the malignant behaviors of lung cancer $(3,4)$. Currently, a body of evidence has established the implication of lncRNAs in lung cancer $(5,6)$. For instance, lncRNA HNF1A-AS1 is significantly more highly expressed in lung cancer compared with the matched non-tumor tissues, and its expression level is significantly associated with Tumor-Node-Metastasis (TNM) stage (7), tumor size and lymph node metastasis, leading to a poorer overall survival rate $(8)$.

In previous studies regarding cancer biomarkers screening, several key lncRNAs have been identified to be significantly downregulated using microarray analysis of renal cell carcinoma (RCC) and adjacent non-tumor tissues (9-11). One of these significantly dysregulated lncRNAs was BX357664 (9), which was later predicted by the Coding Potential Assessment Tool to have no protein-coding potential (12). The BX357664 was initially named CR613822, which was implicated to have functional roles in human cancer. The information regarding CR613822 was uploaded to the NCBI nucleotide database but was deleted by the uploader shortly after. Meanwhile, the updated details referred to the BX357664 gene (12). Furthermore, the length of the sequence of BX357664 is 650 nucleotides (nt) (12). By referring to the definition of lncRNA, the present study operated under the assumption that BX357664 is an IncRNA, and thereafter focused on the functional roles of BX357664 in human cancers. Notably, one pioneer study revealed that BX357664 regulated cell proliferation and epithelial-to-mesenchymal transition via inhibition of TGF- $\beta 1 / \mathrm{p} 38 / \mathrm{HSP} 27$ signaling in RCC (13). This observation reinforced the hypothesis that BX357664 may serve a critical role in human carcinogenesis.

At present, the functional roles of BX357664 in human cancer remain largely unknown. As one part of a larger project focusing on lncRNAs in lung cancer, the present study aimed to investigate the roles of BX357664 in lung cancer cell proliferation, migration and apoptosis. Since BX357664 is a 
novel lncRNA, its expression profile was initially determined in clinical lung cancer and in a series of lung cancer cell lines. The gain-of-function and loss-of-function experiments were then investigated in lung cancer cell lines. The results of the present study may provide novel insight into the molecular targeted treatment and diagnosis of lung cancer in a clinical setting.

\section{Materials and methods}

Human samples. The present study was approved by the Ethics Committee of the Central Hospital of Zhuzhou City (Zhuzhou, China) and written informed consent was obtained from all participants. Lung cancer specimens were randomly selected from 100 patients (male:female, 63:37; age range, 45-70 years; median age, 58 years) who had undergone surgery at the Central Hospital of Zhuzhou City. None of the patients had received chemotherapy or radiotherapy prior to surgery. Tumor tissues and their adjacent non-cancerous tissues were dissected from each case and immediately frozen in liquid nitrogen until use for the subsequent RNA extraction.

Cell culture and transfection. The Human lung cancer A549, H1975 and H-125 cell lines were purchased from American Type Culture Collection (Manassas, VA, USA) and applied for reverse transcription-quantitative polymerase chain reaction (RT-qPCR) analysis to detect the expression of BX357664. Another two lung cancer cell lines, 95D and SPC-A-1, and the normal human 293T cell line were commercially available from the Cell Bank of Chinese Academy of Sciences (Beijing, China) and used to investigate the transcript level of BX357664. Due to the special characteristics of $293 \mathrm{~T}$ cells and lack of normal lung epithelia cells, 293T cells were used as a control. All cells were cultured in the DMEM supplemented with $10 \%$ fetal bovine serum (FBS; Gibco; Thermo Fisher Scientific, Inc., Waltham, MA, USA) at $37^{\circ} \mathrm{C}$ in $5 \% \mathrm{CO}_{2}$ atmosphere. When cells grew to a confluence of $80 \%$, the constructed pcDNA3.1 BX357664 plasmid or the vector (pcDNA3.1, an empty plasmid; Invitrogen; Thermo Fisher Scientific, Inc.), the whole sequence of BX357664 were transfected with $100 \mathrm{ng}$ pcDNA-BX357664 empty vector using Lipofectamine ${ }^{\circledR} 2000$ (Invitrogen; Thermo Fisher Scientific, Inc.) by normal PCR, according to the manufacturer's protocols, and ligation (T4 ligase; New England BioLabs, Inc., Ipswich, MA, USA), according to the manufacturer's protocol. Subsequently, BX357664 plasmid was dissolved in water at $1,000 \mathrm{ng} / \mu \mathrm{l}$ and a total of $2 \mu \mathrm{g}$ DNA was transfected using Lipofectamine 2000 , according to the manufacturer's protocols, into a well in six-well plates $\left(1 \times 10^{4}\right.$ cells). After $6 \mathrm{~h}$ transfection, the culture medium was replaced and $48 \mathrm{~h}$ later, subsequent analysis was performed.

$R N A$ extraction and $R T-q P C R$. Total RNA was extracted from clinical tissues and cultured A549 and 95D cells using TRIzol Reagent (Takara Biotechnology Co., Ltd., Dalian, China), according to the manufacturer's protocol. RNA quality and quantity were determined by Nanodrop 2000 (Thermo Fisher Scientific, Inc.). A total of $500 \mathrm{ng}$ RNAs were reversely transcribed into cDNA using the Transcriptor First Strand cDNA Synthesis kit (Takara Biotechnology Co.,
Ltd.) $\left(37^{\circ} \mathrm{C}\right.$ for $15 \mathrm{~min}$ and $85^{\circ} \mathrm{C}$ for $\left.5 \mathrm{sec}\right)$. The expression levels of BX357664 relative to GAPDH control transcripts were calculated by qPCR using the ABI 7900 Fast Real-Time PCR system (SeqGen, Inc., Torrance, CA, USA). The SYBR ${ }^{\circledR}$ Green reagent was purchased from Takara Biotechnology Co., Ltd. and used with the following protocols: $94^{\circ} \mathrm{C}$ for $10 \mathrm{~min}$ followed by 35 cycles of $94^{\circ} \mathrm{C}$ for $5 \mathrm{sec}$ and $60^{\circ} \mathrm{C}$ for $30 \mathrm{sec}$. The primer sequences were as follows: BX357664 forward, 5'-GGCGTGGTTTTGATGGAGTG-3' and reverse, 5'-AGG CTGCAGAGTTGAGATCG-3'; and GAPDH forward, 5'-ACC ACAGTCCATGCCATCAC-3' and reverse, 5'-TCCACCCTG TTGCTGTA-3'. RT-qPCR amplification was performed in triplicate reactions. The transcription level of BX357664 was normalized to that of GAPDH using the $2^{-\Delta \Delta \mathrm{Cq}}$ method (14).

Colony formation assay. A549 and 95D cells (1x10 $/$ well) were seeded into 12 -well plates $24 \mathrm{~h}$ prior to the transfection. Subsequently, A549 and 95D cells were treated with BX357664-expressing plasmid. A total of 500 cells were seeded into a 6 -well plate in each treatment group. The plates were incubated at $37^{\circ} \mathrm{C}$ for two weeks without changing the culture medium. Finally, the colonies were stained with $1 \%$ crystal violet, and images were captured of five randomly selected fields of view. The whole plates were counted manually and statically analyzed.

Cell proliferation determination. MTT assays (Promega Corporation, Madison, WI, USA) were performed to determine cell proliferation abilities according to the manufacturer's protocol. In brief, A549 and 95D cell lines were seeded into 96-well plates at an initial concentration of $5 \times 10^{3}$ cells/well in the DMEM supplemented with 10\% FBS. A549 and 95D cells were transfected with the BX357664 plasmid, as aforementioned. Each experimental group of cells was seeded in sextuplicate and the culture medium was replaced every other day. The purple formazan was dissolved in dimethyl sulfoxide. Cell proliferation was detected for 5 consecutive days. For each checking point, the cell proliferation rate was detected using a TECAN reader (Tecan Group Ltd., Männedorf, Switzerland) at an absorbance of $490 \mathrm{~nm}$.

Cell cycle analysis. Prior to experimentation, A549 and 95D cells were transfected with pcDNA 3.1 vector or a BX357664-expressing plasmid for $48 \mathrm{~h}$, as aforementioned. Next, cells were collected by low speed centrifugation $\left(1,000 \mathrm{x} \mathrm{g}\right.$ at $4^{\circ} \mathrm{C}$ for $\left.5 \mathrm{~min}\right)$ and fixed with cold ethanol (70\%) for $10 \mathrm{~min}$ at $4^{\circ} \mathrm{C}$. The cells were then washed and re-suspended in pre-cold PBS and incubated at $37^{\circ} \mathrm{C}$ for $30 \mathrm{~min}$ with $10 \mathrm{mg} / \mathrm{ml}$ RNase and $1 \mathrm{mg} / \mathrm{ml}$ propidium iodide (PI; Sigma-Aldrich; Merck KGaA, Darmstadt, Germany). The percentage of cells in each cell cycle phase was determined with a flow cytometer using the Cell Quest acquisition software (Pro version; BD Biosciences, Franklin Lakes, NK, USA).

Transwell assay. A549 and 95D cells were transfected with BX357664-expressing plasmid for $48 \mathrm{~h}$ as described earlier. Subsequently, cells were harvested with serum-free medium and mixed gently, and then $150 \mu \mathrm{l}$ cell suspension $\left(6 \times 10^{4}\right.$ cells) was seeded into the upper chamber $(8 \mu \mathrm{m}$; Corning Incorporated, Corning, NY, USA), while the lower 
A

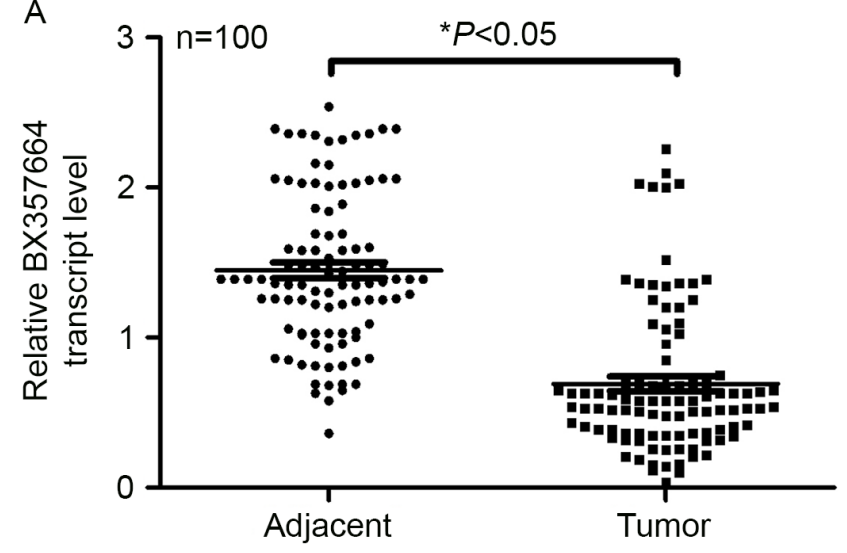

B

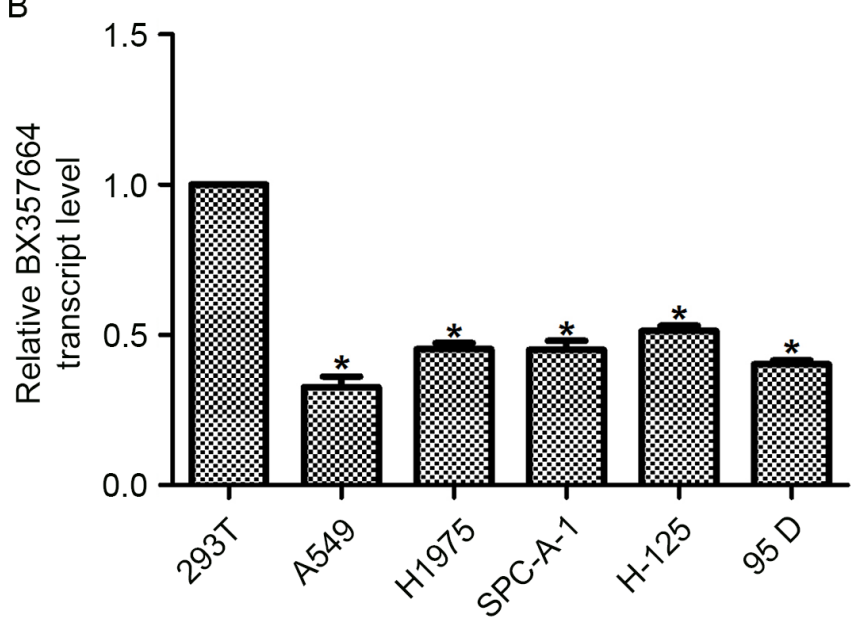

Figure 1. BX357664 is downregulated in lung cancer and differentially expressed in lung cancer cell lines. (A) RT-qPCR analysis of the transcription levels of BX357664 in 100 tumor tissues and their adjacent non-tumor tissues. (B) RT-qPCR analysis of the transcription levels of BX357664 in 5 lung cancer cell lines. The $293 \mathrm{~T}$ cells were cultured simultaneously as controls. ${ }^{*} \mathrm{P}<0.05$ vs. $293 \mathrm{~T}$. RT-qPCR, reverse transcription-quantitative polymerase chain reaction.

chamber was filled with $600 \mu 1$ DMEM supplemented with $10 \%$ FBS. Following incubation for $24 \mathrm{~h}$ at $37^{\circ} \mathrm{C}$, cells in each group were fixed with ice-cold methanol (100\%) for $5 \mathrm{~min}$ at room temperature and stained with $1 \%$ crystal violet for $5 \mathrm{~min}$ at room temperature. Following washing with PBS, cells on the upper surface of the chamber were gently cleaned by a cotton swab. Images of the cells on the lower surface of the membrane were captured and the number of cells was counted under a light microscope (x200; Nikon Corporation, Tokyo, Japan) with five randomly selected fields of view. For cell invasion assays, the membrane was pre-coated with Matrigel (Corning Incorporated, Corning, NY, USA) for $6 \mathrm{~h}$ at $37^{\circ} \mathrm{C}$.

Wound healing assay. The A549 and 95D cell lines were seeded into 6-well plates $\left(3 \times 10^{4}\right.$ cells/well) and treated with the corresponding plasmids for $48 \mathrm{~h}$ at $37^{\circ} \mathrm{C}$ as aforementioned. After the cells had formed a confluent monolayer, the linear wound of the cellular monolayer was created by scratching a straight line through the middle of the plate using a sterile plastic pipette tip $(10 \mu \mathrm{l})$. Cells were washed with PBS and images were captured immediately. Following incubation for $24 \mathrm{~h}$ at $37^{\circ} \mathrm{C}$, images of cells were captured again (Nikon Corporation), and wound closure was calculated in each group.

Statistical analysis. Data were calculated with SPSS 19.0 (IBM Corp., Armonk, NY, USA) and expressed as the mean \pm standard deviation. Differences were evaluated using paired Student's t-test or one-way analysis of variance, followed by the Student-Newman-Keuls post hoc test. $\mathrm{P}<0.05$ was considered to indicate a statistically significant difference.

\section{Results}

BX357664 is downregulated in lung cancer and differentially expressed in lung cancer cell lines. In the present study, the expression profile of BX357664 was initially examined. In the 100 samples collected, RT-qPCR analysis revealed that the average transcription level of BX357664 in the tumor tissues was significantly lower, making up approximately half of that in the adjacent non-tumor tissues $(\mathrm{P}<0.05$; Fig. $1 \mathrm{~A}$ and $\mathrm{B})$. The expression of BX357664 in the 100 lung cancer cases was then classified into low (less than the mean value) and high (higher than the mean value), and was statistically analyzed using clinical categories. It was identified that expression of BX357664 was significantly associated with tumor size, distant metastasis and TNM stage $(\mathrm{P}<0.001)$; however, BX357664 expression was not significantly associated with other examined parameters, including age, marriage status, symptoms and lymph node metastasis (Table I), implying that BX357664 may be associated with the aggressive behaviors observed in lung cancer. Following culture of a series of lung cancer cell lines and the control 293T cells, it was further demonstrated that the relative transcription level of BX357664 was significantly lower in the lung cancer cell lines than in the control 293T cells. Notably, BX357664 had even lower transcription levels in the relatively more aggressive A549 and 95D cell lines. The less aggressive H1975 and H-125 cell lines exhibited a higher expression of BX357664 than the A549 and 95D cell lines. Taken together, these results suggested that BX357664 is downregulated in lung cancer and is differentially expressed in lung cancer cell lines. The A549 and 95D cell lines were selected for subsequent plasmid transfection.

Overexpression of BX357664 in A549 and 95D cells inhibits cell proliferation in vitro. Next, the BX357664 expression plasmid was transfected into A549 and 95D cells. Following transfection, the vector plasmid did not cause any significant changes in the transcription level of BX357664. By contrast, the expression plasmid increased the transcription of BX357664 by nearly 3-fold in A549 and 95D cells (Fig. 2A). These data indicated the high efficiency of the expression plasmid. Colony formation assays were then performed using these gene reconstructs. It was revealed that only $\sim 68$ colonies were formed in A549 cells following overexpression of BX357664, causing a $58 \%$ decrease, compared with the control A549 cells. Similarly, the average number of colonies in BX357664-overexpressing $95 \mathrm{D}$ cells was 70 , which was in contrast with the 138 colonies formed by control 95D cells (Fig. 2B).

In the MTT assay, the proliferative rates were significantly decreased by overexpression of BX357664 on day 4, and on day 5, a 28.6 and $26.3 \%$ decrease in cell numbers was observed 
Table I. Association between BX357664 and clinical variables among 100 patients with lung cancer.

\begin{tabular}{|c|c|c|c|c|}
\hline \multirow[b]{2}{*}{ Variables } & \multirow[b]{2}{*}{ No. $(\%)$} & \multicolumn{2}{|c|}{ Expression of BX357664 } & \multirow[b]{2}{*}{ P-value } \\
\hline & & Low $(\mathrm{n}=60)$ & $\operatorname{High}(\mathrm{n}=40)$ & \\
\hline Age, years & & & & 0.504 \\
\hline$<40$ & $23(23)$ & $8(8)$ & $15(15)$ & \\
\hline $40-55$ & $34(33)$ & $21(21)$ & $13(13)$ & \\
\hline$>55$ & $43(43)$ & $31(31)$ & $13(13)$ & \\
\hline Marriage status & & & & 0.525 \\
\hline Single & $15(15)$ & $7(7)$ & $8(8)$ & \\
\hline Married & $64(64)$ & $45(45)$ & $20(20)$ & \\
\hline Divorced/separated & $21(21)$ & $9(9)$ & $12(12)$ & \\
\hline Presenting symptoms & & & & 0.652 \\
\hline Painless lump & $35(35)$ & $24(24)$ & $11(11)$ & \\
\hline Painful lump & $30(30)$ & $18(18)$ & $12(12)$ & \\
\hline Atypical symptoms & $35(35)$ & $18(18)$ & $17(17)$ & \\
\hline Tumor size $(\mathrm{T})$ & & & & $<0.001^{\mathrm{a}}$ \\
\hline $\mathrm{T} 1(\leq 2 \mathrm{~cm})$ & $17(17)$ & $1(1)$ & $16(16)$ & \\
\hline $\mathrm{T} 2(>2 \mathrm{~cm}-<5 \mathrm{~cm})$ & $23(23)$ & $5(5)$ & $18(18)$ & \\
\hline $\mathrm{T} 3(\geq 5 \mathrm{~cm})$ & $19(19)$ & $15(15)$ & $4(4)$ & \\
\hline T4 (any size with distant metastasis) & $41(41)$ & $39(39)$ & $2(2)$ & \\
\hline Lymph node metastasis & & & & 0.780 \\
\hline No & $45(45)$ & $29(29)$ & $16(16)$ & \\
\hline $\mathrm{N} 1+$ & $55(55)$ & $31(31)$ & $24(24)$ & \\
\hline Distant metastasis & & & & $<0.001^{\mathrm{a}}$ \\
\hline M0 & $58(58)$ & $24(24)$ & $34(34)$ & \\
\hline M1 & $42(42)$ & $36(36)$ & $6(6)$ & \\
\hline TNM stage & & & & $<0.001^{\mathrm{a}}$ \\
\hline $\mathrm{I} / \mathrm{II}$ & $35(35)$ & $5(5)$ & $30(30)$ & \\
\hline III/IV & $65(65)$ & $55(55)$ & $10(10)$ & \\
\hline
\end{tabular}

${ }^{\mathrm{a}} \mathrm{P}<0.001$. TNM, Tumor-Node-Metastasis. P-value was calculated with SPSS software.

in A549 cells (Fig. 2C) and 95D cells (Fig. 2D), respectively. These data suggested that BX357664 inhibits lung cancer cell proliferation.

BX357664 regulates cell cycle progression in lung cancer cells. Cell cycle analysis revealed that the cell percentage in the G0/G1 phase was increased by $\sim 10 \%$, while the cell percentages in the $\mathrm{S}$ and $\mathrm{G} 2 / \mathrm{M}$ phases were decreased in BX357664-overexpressing A549 cells (Fig. 3A). Similarly, Overexpression of BX357664 in 95D cells also resulted in cell cycle arrest in the G0/G1 phase, as evidenced by the increased percentage of cells in the G0/G1 phase (Fig. 3B). These observations suggested that BX357664 regulates cell cycle progression in lung cancer cells.

Overexpression of BX357664 in A549 and 95D cells inhibits cell metastasis in vitro. In light of the association of BX357664 expression with TNM staging and distant metastasis (Table I), the effects of BX357664 on cell migration and invasion capacities were further evaluated. In the Transwell migration assay, a significant number of A459 and 95D cells migrated to the lower surface of the membrane, which was evidenced by the crystal violet staining (Fig. 4A and B). In the BX357664-overexpressing cells, only an average of 208 A549 cells migrated, which was in contrast to the 408 control A549 cells. A similar result was also observed in the invasion assay in BX357664-overexpressing 95D cells (Fig. 4C). The potential of cells to invade through the membrane was inhibited by $>65 \%$ when $95 \mathrm{D}$ cells were transfected with the BX357664-expressing plasmid (Fig. 4D). The multi-cell lines-based observations suggested that BX357664 may function as a negative regulator of cell metastasis in lung cancer.

In the wound healing assay, when BX357664 was overexpressed, the wound recovery was significantly decreased in A549 (Fig. 5A and B) and 95D (Fig. 5C and D) cells. In particular, the overexpression of BX357664 in A549 cells inhibited cell migration by $52 \%$ since the area of wound closure was decreased (Fig. 5B). Likewise, the overexpression of BX357664 in 95D cells resulted in similar effects (Fig. 5D). The wound recovery rates corroborated the Transwell migration and invasion results. 


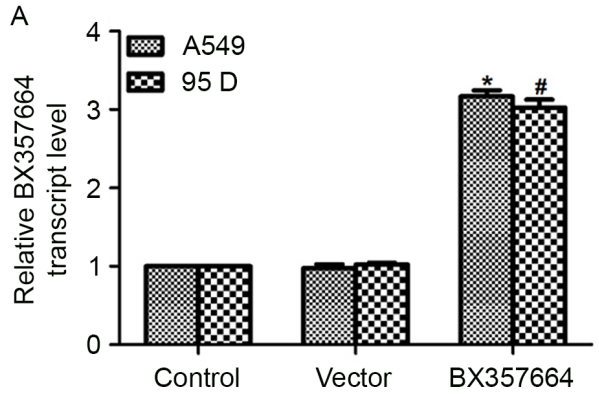

C

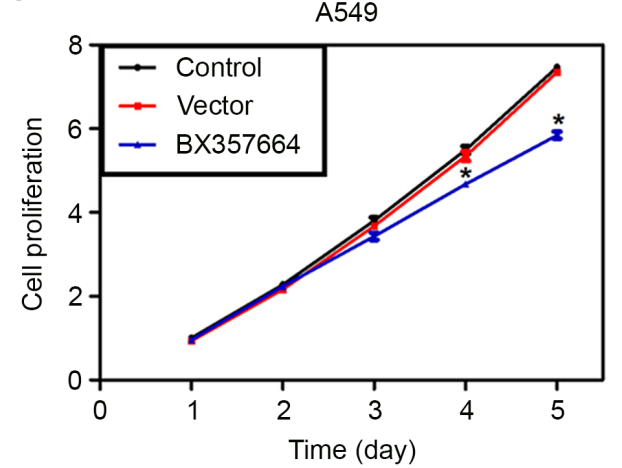

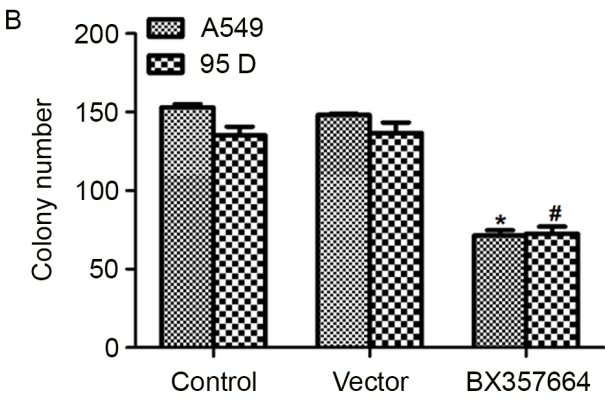

D

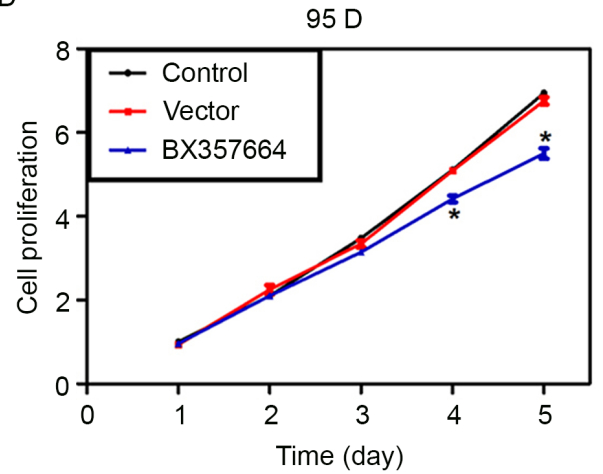

Figure 2. Overexpression of BX357664 inhibits cell colony formation and cell proliferation. (A) Transfection efficiency of the expression plasmid of BX357664 was examined in A549 and 95D cells. (B) Colony formation assay was performed in control and plasmid-transfected A549 and 95D cells. The colonies were counted and the average colony was calculated and compared. "P $<0.05$ vs. control. (C) Following transfection of BX357664 plasmids, cell proliferation was monitored for 5 consecutive days in A549 cells. (D) Following transfection of BX357664 plasmids, cell proliferation was monitored for 5 consecutive days in 95D cells. ${ }^{*} \mathrm{P}<0.05$ vs. control.

\section{Discussion}

LncRNAs are a class of non-coding RNAs with a length of $>200$ nucleotides (15). It has been demonstrated that the aberrant expression of lncRNAs may contribute toward carcinogenesis and specific lncRNAs have been identified as oncogenes or tumor suppressor genes (16-18). The present study investigated the role of a novel lncRNA, BX357664, in the behaviors of lung cancer. BX357664 was implicated to serve functional roles in human cancer (12), including renal cell carcinoma (RCC) (9). The upregulation of BX357664 was demonstrated to reduce the migration, invasion and proliferation capabilities of RCC cells (13). These data suggested that lncRNAs serve a functional role in solid tumors. The study of BX357664 in human lung cancer would enhance the present understanding of lung carcinogenesis and provide novel evidence for the diagnosis and treatment of lung cancer.

In the present study, it was initially demonstrated that BX357664 was downregulated in clinical lung cancer tissues and in a series of lung cancer cell lines, which was consistent with previous reports that BX357664 was significantly deregulated by a microarray analysis in lung cancer (9) and in RCC tissues (13). BX357664 expression was also demonstrated to be significantly associated with clinical parameters, including tumor size, distant metastasis and TNM staging. This was corroborated by the results of the present study, where the expression of BX357664 was observed to be higher in less aggressive lung cancer A549 and 95D cell lines, whereas BX357664 expression was lower in the more aggressive H1975 and H-125 cell lines, implying

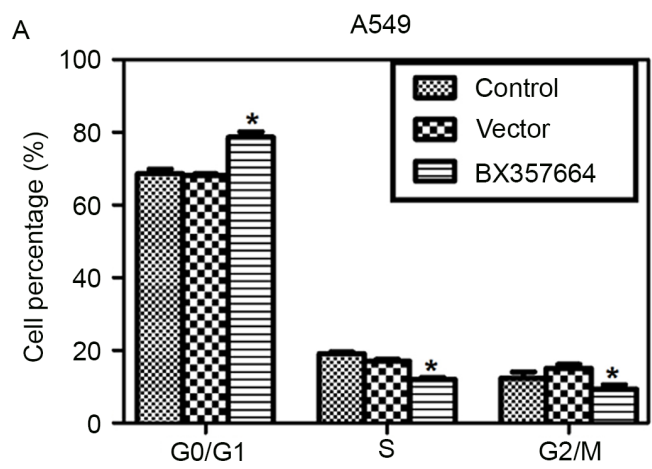

B

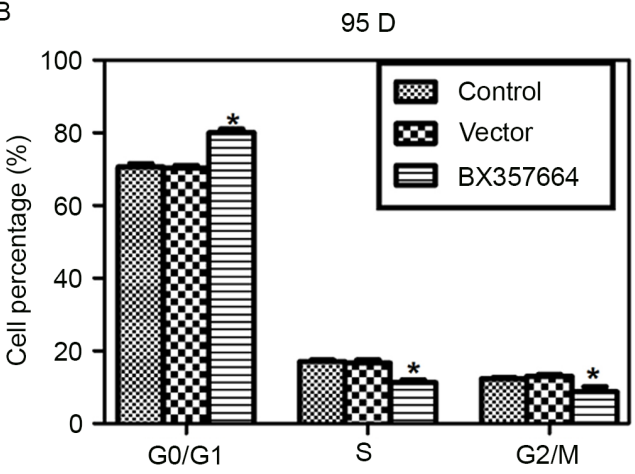

Figure 3. BX357664 regulates cell cycle progression in lung cancer cells. (A) Cell cycle analysis revealed that the percentage of cells in the G0/G1 phase was increased by $\sim 10 \%$, while the percentage of cells in the $\mathrm{S}$ and G2/M phases was decreased in BX357664-overexpressing A549 cells. (B) Cell cycle analysis revealed that the percentage of cells in the G0/G1 phase was increased by $\sim 10 \%$, while the percentage of cells in the $\mathrm{S}$ and G2/M phases was decreased in BX357664-overexpressing 95D cells. ${ }^{*} \mathrm{P}<0.05$ vs. control cells. 
A

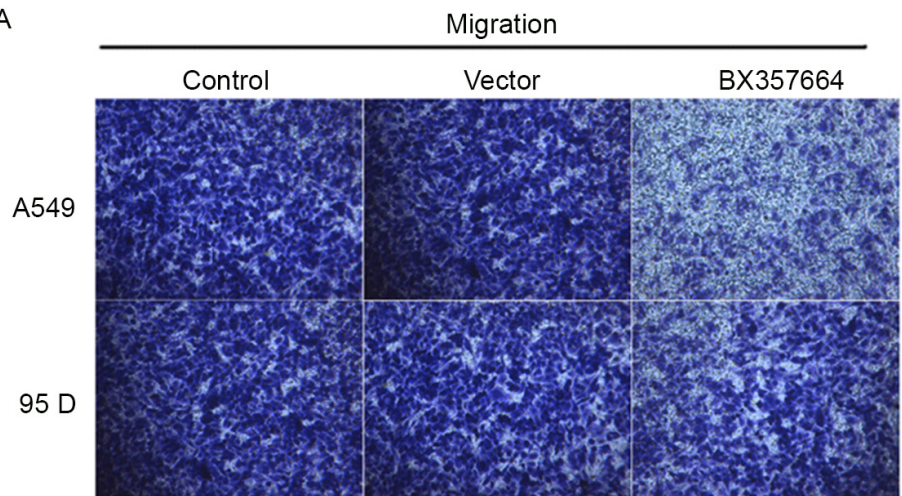

C

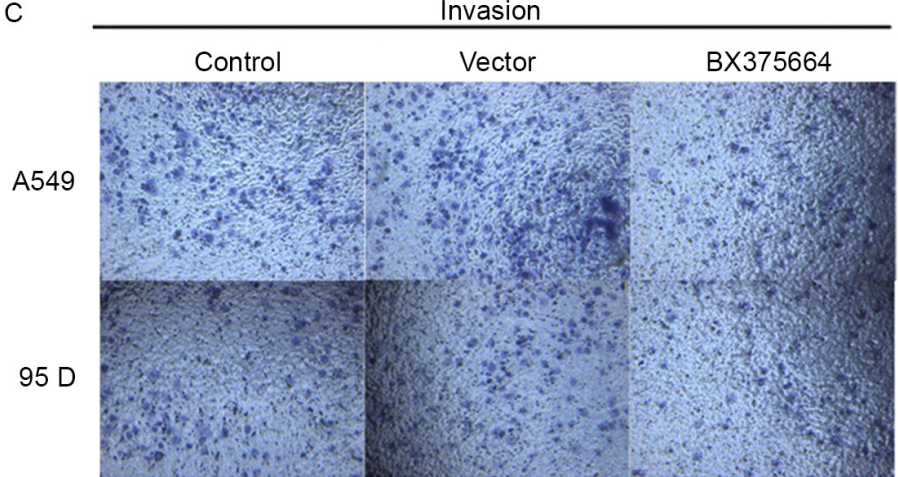

B

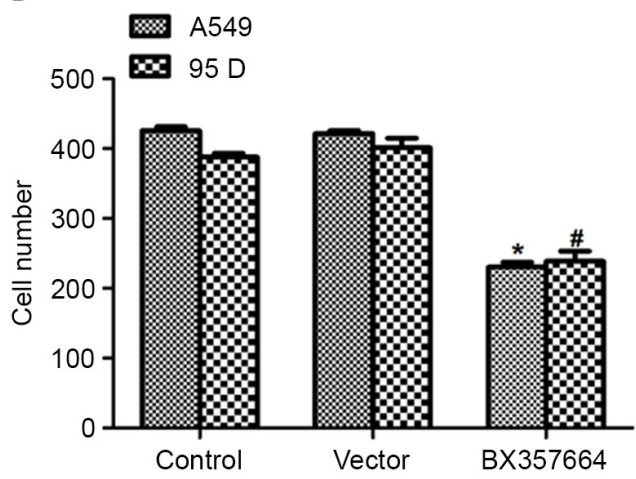

D

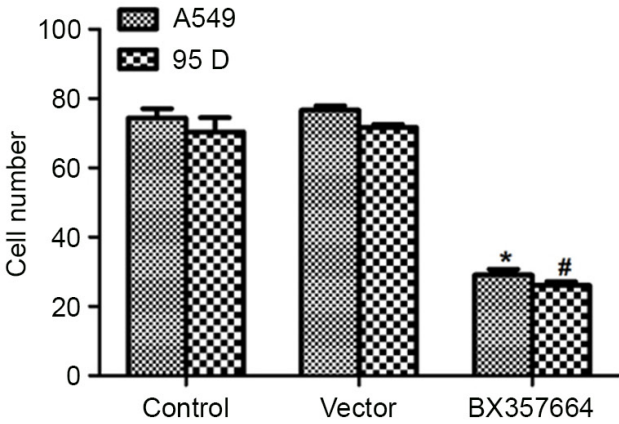

Figure 4. Overexpression of BX357664 inhibits cell migration and invasion in vitro. (A) Transwell migration was performed in control and BX357664-overexpressing A549 and 95D cells. (B) Quantification of cell migration demonstrated that an overexpression of BX357664 inhibited cell migration. (C) Invasion assays were also performed in control and BX357664-overexpressing A549 and 95D cells. (D) Quantification of cell invasion assay. Following migration, transmigrated cells under the lower surface of membrane were stained with crystal violet. Cells in each treatment group were counted. Magnification, $\mathrm{x} 200 .{ }^{*} \mathrm{P}<0.05$ vs. control cells in A549 cells. ${ }^{~} \mathrm{P}<0.05$, vs. control in $95 \mathrm{D}$ cells.

A

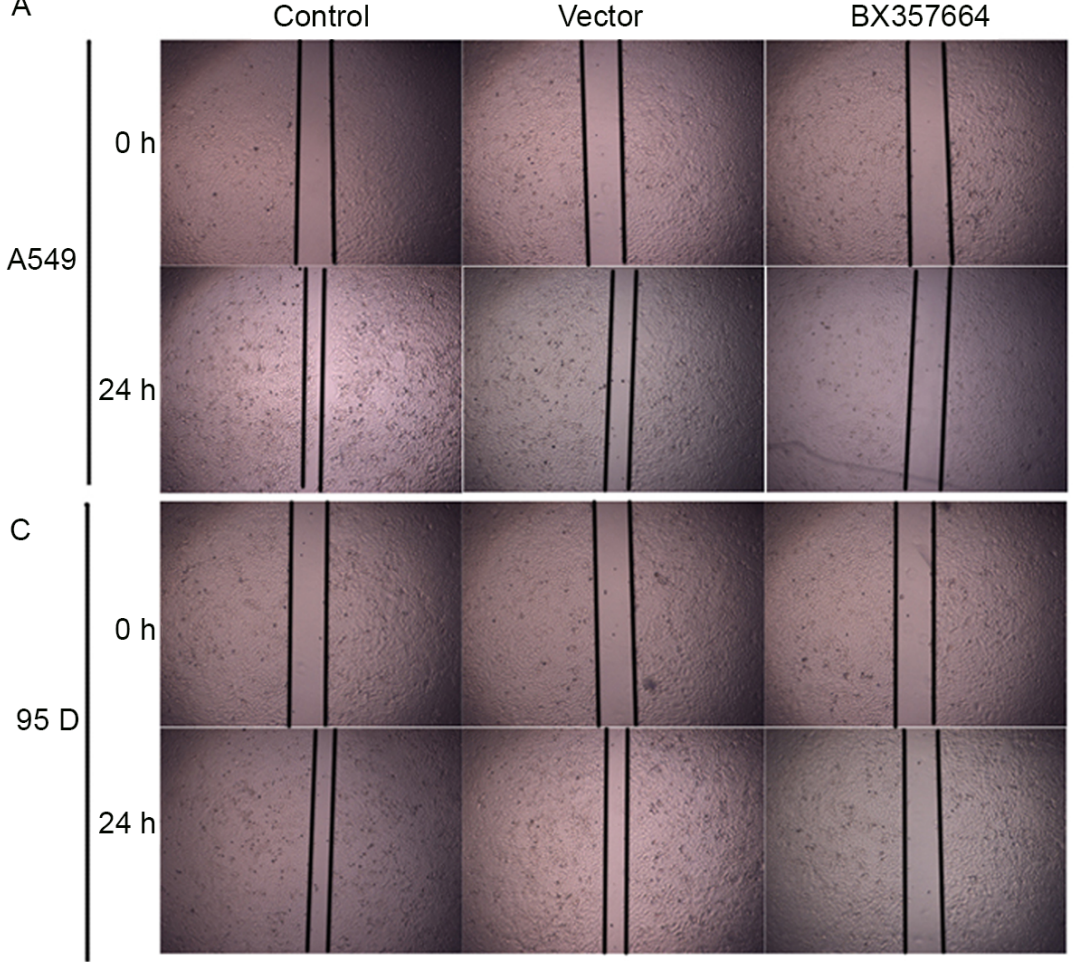

B

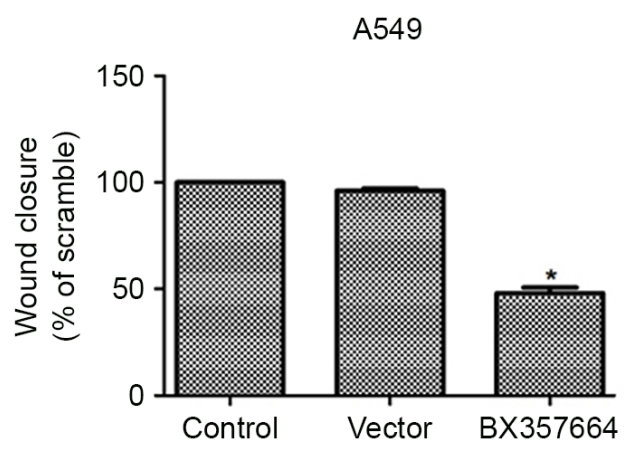

D

$95 \mathrm{D}$

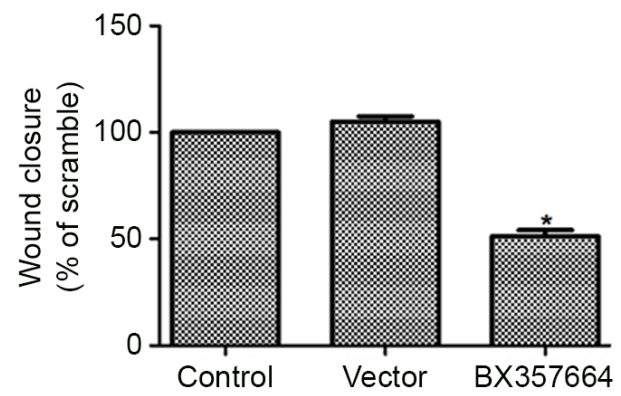

Figure 5. Overexpression of BX357664 inhibits wound recovery processes in vitro. (A) Representative images of A549 cells in wound-healing assays. (B) Quantification of wound-healing assays in A549 cells treated with BX357664-expressing plasmid. (C) Representative images of 95D cells in wound-healing assays. (D) Quantification of wound-healing assays in 95D cells treated with BX357664-expressing plasmid. Magnification, $\mathrm{x} 200$. " $\mathrm{P}<0.05$ vs. control cells in each cell line. 
that BX357664 may be a determining factor in the aggressiveness of lung cancer cells. Therefore, the A549 and 95D cell lines were utilized for the subsequent gain-of-function experiments. It was demonstrated that the overexpression of BX357664 inhibited the cell clonogenic potential and proliferative abilities.

Cell cycle arrest is a hallmark of carcinogenesis (19). Based on the cell viability assays, cell cycle progression was assessed. As a consequence of BX357664 overexpression in A549 and 95D cells, cell percentages in the G0/G phase were significantly increased. These results suggested that BX357664 inhibits cell proliferation in lung cancer cells. In addition, it was further demonstrated that overexpression of BX357664 inhibited cell migration and invasion abilities in A549 and 95D cells. Wound recovery abilities were accordingly impaired. Together with cell viability assays, the multi-cell lines-based observations suggested that BX357664 serves a crucial role in cell proliferation and metastasis in lung cancer. Overexpression of BX357664 may be a novel therapeutic strategy for the treatment of lung cancer.

It would be of scientific value to investigate the mechanisms underlying BX357664-mediated biological behaviors in lung cancer. However, a limitation of the present study is that the expression of lncRNA BX357664 within each pathological subtype of lung cancer was not analyzed $(20,21)$. Whether lncRNA BX357664 has any distinct functional roles in different pathological subtypes remains to be elucidated. This limitation requires the collection of substantial cases categorized as different pathological subtypes. Our future studies will address this, as well as attempting to investigate the mechanisms underlying the functions of BX357664 in human cancer.

In conclusion, the results of the present study suggested that the IncRNA BX357664 is a critical inhibitor of cell proliferation and metastasis, and an inducer of cell apoptosis in lung cancer. The results may also provide novel insight into the clinical diagnosis of lung cancer and suggested that overexpression of BX357664 may be a promising therapeutic strategy for the treatment of lung cancer.

\section{Acknowledgements}

Not applicable.

\section{Funding}

No funding was received.

\section{Availability of data and materials}

All data generated or analyzed during the present study are included in this published article.

\section{Authors' contributions}

SHX and GXL performed the majority of the experiments. LQ analyzed the data and revised the manuscript. YF designed the project, analyzed the data and wrote the manuscript.

\section{Ethics approval and consent to participate}

The present study was approved by the Ethics Committee of the Central Hospital of Zhuzhou City (Zhuzhou, China) and written informed consent was obtained from all participants.

\section{Patient consent for publication}

All patients showed their full intention for publication and provided written informed consent.

\section{Competing interests}

The authors declare that they have no competing interests.

\section{References}

1. Liu J, Wan L, Lu K, Sun M, Pan X, Zhang P, Lu B, Liu G and Wang Z: The long noncoding RNA MEG3 contributes to cisplatin resistance of human lung adenocarcinoma. PLoS One 10: e0114586, 2015.

2. Siegel R, Naishadham D and Jemal A: Cancer statistics, 2013. CA Cancer J Clin 63: 11-30, 2013.

3. Chen J, Wang R, Zhang K and Chen LB: Long non-coding RNAs in non-small cell lung cancer as biomarkers and therapeutic targets. J Cell Mol Med 18: 2425-2436, 2014.

4. Zhang L, He T, Yan Y, Zhang Y, Zhou X, Huang P, Kong Y, Xie M, Zhang L, Sun Q, et al: Expression and clinical significance of the novel long noncoding RNA ZNF674-AS1 in human hepatocellular carcinoma. Biomed Res Int 2016: 608914, 2016.

5. Yu J, Fang Q and Meng S: Knockdown of long noncoding RNA ENST457720 inhibits proliferation of non-small cell lung cancer cells in vitro and in vivo. Oncol Res, Mar 1, 2018 (Epub ahead of print).

6. Yu W, Peng W, Jiang H, Sha H and Li J: LncRNA HOXA11-AS promotes proliferation and invasion by targeting miR-124 in human non-small cell lung cancer cells. Tumour Biol 39: $1010428317721440,2017$.

7. Chansky K, Detterbeck FC, Nicholson AG, Rusch VW, Vallières E, Groome P, Kennedy C, Krasnik M, Peake M, Shemanski L, et al: The IASLC lung cancer staging project: External validation of the revision of the TNM stage groupings in the eighth edition of the TNM classification of lung cancer. J Thorac Oncol 12: 1109-1121, 2017.

8. Wu Y, Liu H, Shi X, Yao Y, Yang W and Song Y: The long non-coding RNA HNF1A-AS1 regulates proliferation and metastasis in lung adenocarcinoma. Oncotarget 6: 9160-9172, 2015.

9. Qin C, Han Z, Qian J, Bao M, Li P, Ju X, Zhang S, Zhang L, Li S, Cao Q, et al: Expression pattern of long non-coding RNAs in renal cell carcinoma revealed by microarray. PLoS One 9: e99372, 2014.

10. Yang Y, Zhao L, Lei L, Lau WB, Lau B, Yang Q, Le X, Yang H, Wang C, Luo Z, et al: LncRNAs: The bridge linking RNA and colorectal cancer. Oncotarget 8: 12517-12532, 2017.

11. Liu T, Zhang X, Gao S, Jing F, Yang Y, Du L, Zheng G, Li P, $\mathrm{Li} \mathrm{C}$ and Wang C: Exosomal long noncoding RNA CRNDE-h as a novel serum-based biomarker for diagnosis and prognosis of colorectal cancer. Oncotarget 7: 85551-85563, 2016.

12. Wang L, Park HJ, Dasari S, Wang S, Kocher JP and Li W: CPAT: Coding-Potential Assessment Tool using an alignment-free logistic regression model. Nucleic Acids Res 41: e74, 2013.

13. Liu Y, Qian J, Li X, Chen W, Xu A, Zhao K, Hua Y, Huang Z, Zhang J, Liang C, et al: Long noncoding RNA BX357664 regulates cell proliferation and epithelial-to-mesenchymal transition via inhibition of TGF- $\beta 1 / \mathrm{p} 38 / \mathrm{HSP} 27$ signaling in renal cell carcinoma. Oncotarget 7: 81410-81422, 2016.

14. Livak KJ and Schmittgen TD: Analysis of relative gene expression data using real-time quantitative PCR and the 2(-Delta Delta C(T)) method. Methods 25: 402-408, 2001.

15. Rinn JL: lncRNAs: Linking RNA to chromatin. Cold Spring Harb Perspect Biol 6: pii: a018614, 2014.

16. Guo X, Xia J and Deng K: Long non-coding RNAs: Emerging players in gastric cancer. Tumour Biol 35: 10591-10600, 2014. 
17. Zhang H, Guo Y, Song Y and Shang C: Long noncoding RNA GAS5 inhibits malignant proliferation and chemotherapy resistance to doxorubicin in bladder transitional cell carcinoma. Cancer Chemother Pharmacol 79: 49-55, 2017.

18. Lee J, Jung JH, Chae YS, Park HY, Kim WW, Lee SJ, Jeong JH and Kang SH: Long noncoding RNA snaR regulates proliferation, migration and invasion of triple-negative breast cancer cells. Anticancer Res 36: 6289-6295, 2016.

19. Sherr CJ: Cancer cell cycles. Science 274: 1672-1677, 1996.

20. Seki N, Eguchi K, Kaneko M, Ohmatsu H, Kakinuma R, Matsui E, Kusumoto M, Tsuchida T, Nishiyama $\mathrm{H}$ and Moriyama $\mathrm{N}$ : Stage-size relationship in long-term repeated CT screening for lung cancer: Anti-lung cancer association project. J Clin Oncol 27: S1540, 2009.
21. Nitsche U, Stangel D, Pan Z, Schlitter AM, Esposito I, Regel I, Raulefs S, Friess H, Kleeff J and Erkan M: Periostin and tumor-stroma interactions in non-small cell lung cancer. Oncol Lett 12: 3804-3810, 2016.

(i) (3) This work is licensed under a Creative Commons Attribution-NonCommercial-NoDerivatives 4.0 International (CC BY-NC-ND 4.0) License. 